\title{
Estructura de la comunidad de peces en el sistema Candelaria-Panlau, Campeche, México
}

\author{
Luis Amado Ayala-Pérez ${ }^{1}$, Obed Agustín Avilés-Alatriste ${ }^{1}$ y José Luis Rojas-Galaviz ${ }^{2}$ \\ 1 Departamento "El Hombre y su Ambiente". Universidad Autónoma Metropolitana Xochimilco. Calz. del Hueso 1100 Col. \\ Villaquietud. Coyoacán 04960 México, D.F. Fax: 72354 69, e-mail luayala@cueyatl.uam.mx \\ 2 Centro EPOMEX. Av. Agustín Melgar y Juan de la Barrera s/n. A.P. 520 Campeche 24030, Campeche, México.
}

Recibido 18-IV-1997. Corregido 2-IV-1998. Aceptado 14-IV-1998.

\begin{abstract}
The environmental and ecological patterns of the fish community in Candelaria-Panlau system (Mexico) are described. Parameters of the water temperature, salinity and transparency, and density, biomass and diversity of the fish community were analyzed. Between April 1993 and April 1994 five stations were sampled monthly. The fish community had 50 species (7926 individuals, $144.7 \mathrm{~kg}$ of weight). The abundance and diversity values for the fish community were: $0.09 \mathrm{ind} / \mathrm{m}^{2} ; 1.61 \mathrm{~g} / \mathrm{m}^{2} ; 18.25 \mathrm{~g} / \mathrm{ind} ; \mathrm{H} ' \mathrm{n}=1.879 ; \mathrm{J}=0.475$ and $\mathrm{D}=5.681$. The species with ecological dominance were: Cathorops melanopus, Diapterus rhombeus, Anchoa mitchilli, Sphoeroides testudineus, Bairdiella chrysura, $B$. ronchus, Cynoscion arenarius, and $C$. nebulosus. The matrixes of the environmental parameters and of fish abundance, were tested with a factor analysis (principal components extraction method); its graphical representation confirms the dominant species and indicates that salinity, station number five and February are the components that characterize the system and the fish community.
\end{abstract}

Key words: Fish community, Candelaria-Panlau, estuarine ecology, tropical marsh, fish ecology.

En la región de la Laguna de Términos, en el estado de Campeche, se han realizado diversos estudios ecológicos que han permitido identificar el valor de la estructura y función de los sistemas fluvio-lagunares asociados, los cuales constituyen un "puente ecológico" entre la planicie costera y la plataforma continental de la Sonda de Campeche (Vera-Herrera et al. 1988a, Ayala-Pérez et al. 1993, Ayala-Pérez et al. 1995).

Los sistemas fluvio-lagunares son áreas de almacén y transporte de sedimentos, materia orgánica, nutrimentos, detritos y organismos, y están sujetos a una presión ambiental definida principalmente por el volumen de descarga de los ríos, el nivel de marea, la geomorfología, el tipo y abundancia de la vegetación asociada y la temporada climática (Vera-Herrera et al. 1988a).

Funcionan como hábitat de una diversa comunidad biótica, donde la macrofauna acuática más importante la constituyen los peces dadas sus características de abundancia, diversidad y distribución en espacio y tiempo. Estos organismos utilizan de manera programada los distintos hábitats, para desarrollar parte de sus ciclos de vida, unos se consideran recursos pesqueros actuales $o$ potenciales y otros son el vehículo de grandes cantidades de energía, lo cual se traduce en un gran valor ecológico (Soberón-Chávez 1984). 
Es necesario mencionar que los sistemas fluvio-lagunares forman parte de la zona declarada por Decreto del Ejecutivo Federal Mexicano como área natural protegida con el carácter de área de protección de flora y fauna "Laguna de Términos" (Anónimo 1994a).

La primera descripción de las comunidades de peces en los sistemas fluvio-lagunares asociados a la Laguna de Términos la presentan Amezcua-Linares y Yáñez-Arancibia (1980). Estudios más recientes sobre la ecología y biología de peces en cada uno de los sistemas son los siguientes: en Palizada-Del Este destacan Morales (1986), Vera-Herrera et al. (1988b), Rojas-Galaviz et al. (1988), Ayala-Pérez (1989), Fuentes-Yaco (1990), en ChumpamBalchacah, Ayala-Pérez et al. (1996), Ayala-Pérez y Avilés-Alatriste, (en prep.), en el caso del sistema Pom-Atasta sólo se han identificado reportes técnicos de investigación (Aguirre-León et al. 1995); para el sistema Candelaria-Panlau se cuenta con la información de Amezcua-Linares y
Yáñez-Arancibia (1980), Nitsch (1992) y finalmente un análisis de integración para tres sistemas fluvio-lagunares es presentado por AyalaPérez et al. (1993).

Con este marco conceptual, en este documento se describen y analizan los patrones espacio-temporales de los principales parámetros ambientales y de la estructura de la comunidad de peces en el sistema Candelaria-Panlau para un ciclo anual.

\section{MATERIALES Y METODOS}

El sistema Candelaria-Panlau se localiza en la porción Este de la Laguna de Términos y está integrado por las desembocaduras de los ríos Candelaria y Mamantel, la Laguna Panlau y la boca de conexión con la Laguna de Términos denominada Boca de los Pargos (Fig. 1). La cuenca del río Candelaria se localiza en la Península de Yucatán y se ha calculado que tiene

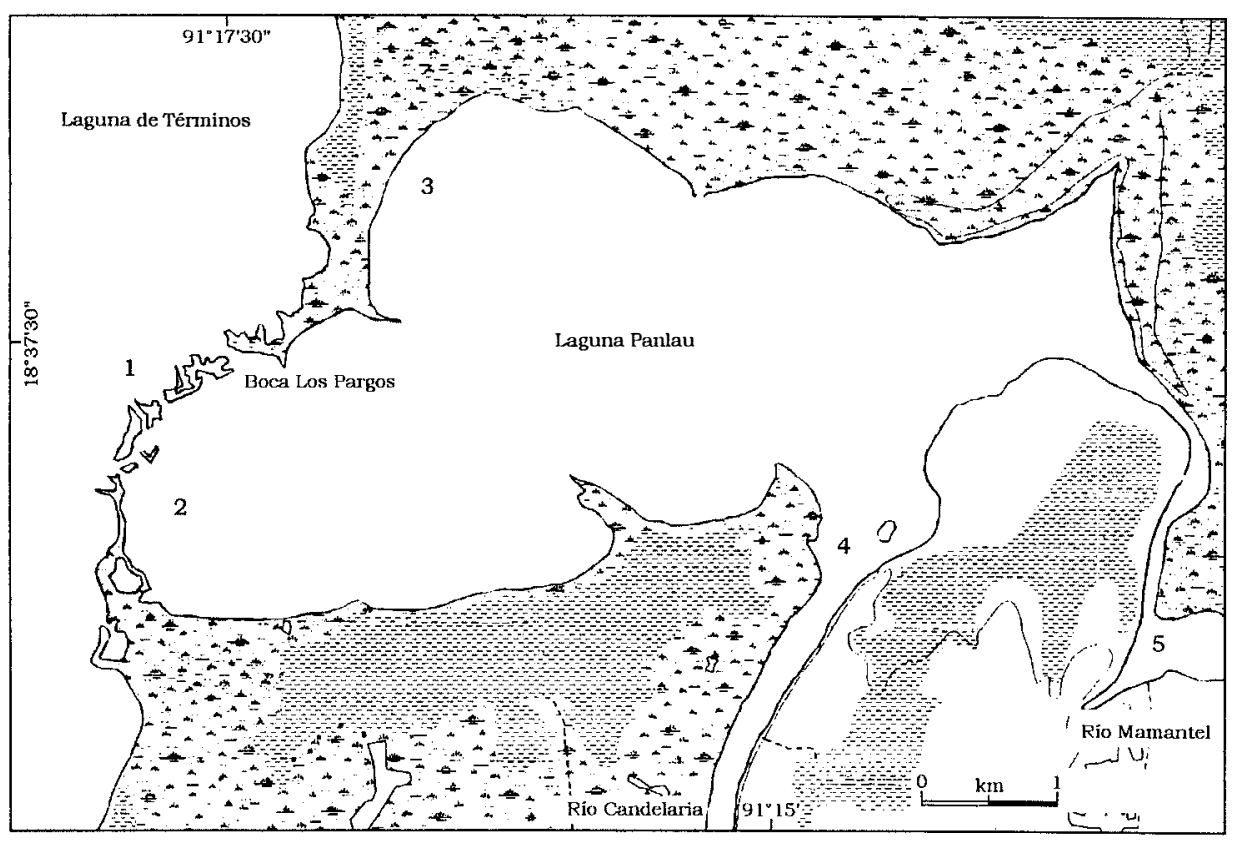

Fig.1. Localización geográfica del sistema Candelaria-Panlau, Campeche, México. La numeración representa la ubicación de las estaciones de muestreo. 
un aporte de $21.5 \mathrm{~m}^{3} / \mathrm{s}$ (Anónimo 1976). La Laguna Panlau tiene un área aproximada de 14 $\mathrm{km}^{2}$, y la cruza de Este a Oeste un camino construido por los Mayas como paso comercial (según habitantes de la región), y que emerge cuando disminuye el nivel del agua. La profundidad de la laguna varía entre $1.0 \mathrm{~m}$ y $3.5 \mathrm{~m}$ con un promedio de $2.0 \mathrm{~m}$. El tipo de marea en la región es mixta-diurna con una pleamar media de $0.18 \mathrm{~m}$ y una bajamar media de $-0.24 \mathrm{~m}$. (Anónimo 1994b). La salinidad del agua oscila entre cinco y 26 unidades y la temperatura entre 28 y $31^{\circ} \mathrm{C}$. El sedimento es limo arcilloso con carbonato de calcio y la vegetación circundante es Rhizophora mangle y Avicennia germinans principalmente. (Amezcua-Linares y YáñezArancibia 1980, Nitsch 1992).

Entre abril de 1993 y abril de 1994 se realizaron muestreos mensuales en cinco estaciones; cuya ubicación se representa en la Fig. 1. Las muestras de peces se obtuvieron con una red de prueba camaronera de $5 \mathrm{~m}$ de largo, $2.5 \mathrm{~m}$ de abertura de trabajo y luz de malla de $2 \mathrm{~cm}$, con tablas de arrastre de $0.8 \times 0.5 \mathrm{~m}$, a bordo de una lancha de $7 \mathrm{~m}$ de eslora con motor fuera de borda de $55 \mathrm{HP}$. El arrastre tuvo una duración de 10 minutos a una velocidad promedio de 2 nudos. Las muestras se almacenaron en bolsas de plástico etiquetadas y se fijaron con una solución de formaldehído al $10 \%(\mathrm{v} / \mathrm{v})$.

Inmediatamente después del arrastre se hicieron las mediciones de temperatura y salinidad del agua en superficie y fondo, además de la transparencia, y temperatura ambiente. El equipo utilizado fue: termómetro de cubeta con precisión de $0.5^{\circ} \mathrm{C}$, salino-termo-conductivímetro YSI con precisión de 0.5 unidades de salinidad, botella van Dorn, sondaleza y disco de Secchi.

La identificación taxonómica de las especies se realizó utilizando literatura especializada: Jordan y Evermann (1886-1900), Hildebrand (1943), Alvarez del Villar (1970), Castro-Aguirre (1978), Hoese y Moore (1977), Fischer (1978), y Reséndez (1981 a y b). El ordenamiento sistemático se hizo de acuerdo con Nelson (1994).
Los peces se midieron con un ictiómetro convencional y se pesaron con una balanza digital de $2.2 \mathrm{~kg}$ de capacidad y $0.1 \mathrm{~g}$ de precisión. El área barrida por el arte de pesca se calculó como el producto de la velocidad de arrastre por el tiempo de operación por la abertura de trabajo de la red. Los parámetros de abundancia se calcularon en términos de densidad (ind $\left./ \mathrm{m}^{2}\right)$, biomasa $\left(\mathrm{g} / \mathrm{m}^{2}\right)$ y peso promedio ( $\left.\mathrm{g} / \mathrm{ind}\right)$, y los de diversidad mediante las expresiones de Shannon y Weaver (1963), Margalef (1969) y Pielou (1966).

Para identificar a las especies con dominio ecológico se siguió el siguiente criterio: integrar a las especies que de una forma equilibrada presenten los valores más altos de abundancia en número de individuos y en peso, de frecuencia de aparición y de amplitud en su distribución. El número total de especies dominantes se definió por la representación del $80 \%$ de la abundancia de la captura total.

El análisis gráfico y de tendencias polinomiales tanto de los parámetros ambientales como ecológicos se realizó con la ayuda del programa Excel. A los parámetros ambientales y de abundancia numérica de la comunidad de peces se les aplicó un análisis de factores con el método de extracción de componentes principales y los resultados se representan gráficamente con la rotación de varianza máxima normalizada, se utilizó el programa STATISTICA (Pielou 1984, Ludwig and Reynolds 1988 y Gauch 1989).

\section{RESULTADOS}

El comportamiento temporal de los parámetros ambientales del sistema Candelaria-Panlau para el periodo abril de 1993-abril de 1994 queda representado por la variación de los parámetros de temperatura, salinidad, transparencia del agua y temperatura ambiente. En las Figs. dos y tres se presentan los valores mínimo, máximo y promedio por mes de cada parámetro. En el caso de la temperatura del agua de superficie, los valores más altos se presentaron en mayo con un promedio de $30.7^{\circ} \mathrm{C}$ y los más bajos en enero con un promedio de $23.9^{\circ} \mathrm{C}$. Un 
modelo polinomial de tercer orden $(\mathrm{y}=$ $\left.0.0346 \mathrm{x}^{3}-0.7307 \mathrm{x}^{2}+3.9044+24.503\right)$ se ajustó a este comportamiento con una correlación $\mathrm{R}^{2}=0.7418$. Con un comportamiento similar, la temperatura del agua de fondo presentó los valores más altos en mayo con un promedio de $30.5^{\circ} \mathrm{C}$ y el más bajo en enero con un promedio de $24.0^{\circ} \mathrm{C}$. También un modelo polinomial de tercer orden $\left(\mathrm{y}=0.0335 \mathrm{x}^{3}-0.7081 \mathrm{x}^{2}+3.787+\right.$ 24.676) describe este comportamiento con una correlación $\mathrm{R}^{2}=0.7435$ (Fig.2). En el mismo sentido, el comportamiento de la salinidad del agua tanto en superficie como en fondo muestra una tendencia temporal donde los valores más altos se presentaron en mayo con un promedio de 18.2 UPS (Unidades Prácticas de Salinidad) y 18.3 UPS respectivamente y los más bajos se observaron en noviembre con promedio de 2.36 UPS y 5.36 UPS. El modelo polinomial que describe este comportamiento para superficie es: $\mathrm{y}=0.0517 \mathrm{x}^{3}-0.9772 \mathrm{x}^{2}+1.8017+15.066$ con una correlación $\mathrm{R}^{2}=0.8431$, y para fondo $\mathrm{y}=0.0701 \mathrm{x}^{3}-1.2299 \mathrm{x}^{2}+4.7843+12.574 \mathrm{con}$ una correlación $\mathrm{R}^{2}=0.8704$ (Fig. 2).
La temperatura ambiente muestra un patrón donde los valores más altos se presentaron en mayo con un promedio de $33^{\circ} \mathrm{C}$ y los más bajos en enero con un promedio de $23{ }^{\circ} \mathrm{C}$. Finalmente, la transparencia del agua muestra un ámbito de variación entre 60.4 y $32.7 \%$, estos extremos se presentaron en septiembre y octubre respectivamente (Fig. 3).

La representación espacial del comportamiento de estos parámetros ambientales se presenta en las Figs. cuatro y cinco. La temperatura del agua en los dos niveles osciló entre 27.4 ${ }^{\circ} \mathrm{C}$ y $28.0^{\circ} \mathrm{C}$, los valores máximos se registraron en la estación cinco. De igual forma, la salinidad en superficie y en fondo presentó valores entre 1.6 UPS y 27.0 UPS, los valores máximos se registraron en la estación uno y los mínimos en la estación cuatro (boca del río Candelaria) (Fig. 4). La temperatura ambiente muestra valores promedio de entre $26.5^{\circ} \mathrm{C}$ en la estación uno y $29.1{ }^{\circ} \mathrm{C}$ en la estación cinco. Finalmente, los valores promedio de transparencia oscilan entre $35.3 \%$ para la estación cinco y $68.7 \%$ para la estación tres (Fig. 5).
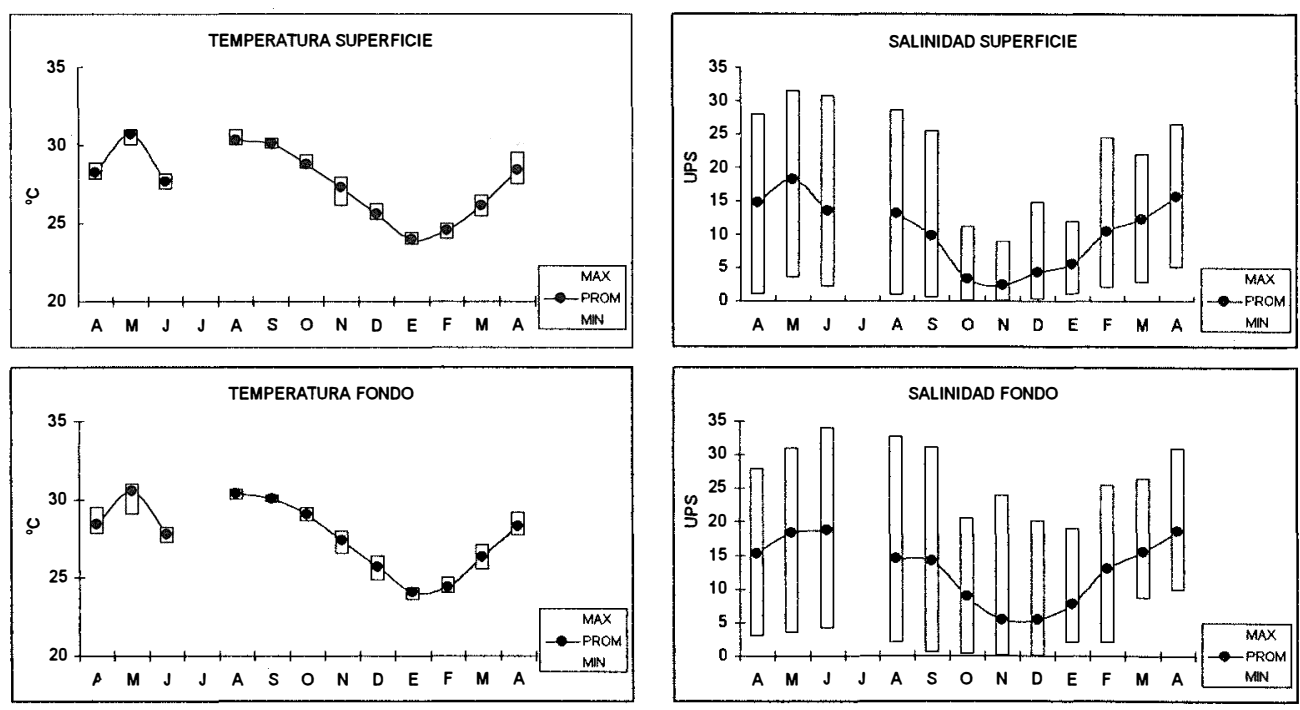

Fig.2. Variación temporal de los parámetros de temperatura y salinidad en el sistema Candelaria-Panlau. Se presentan los valores mínimo, máximo y promedio por mes. 

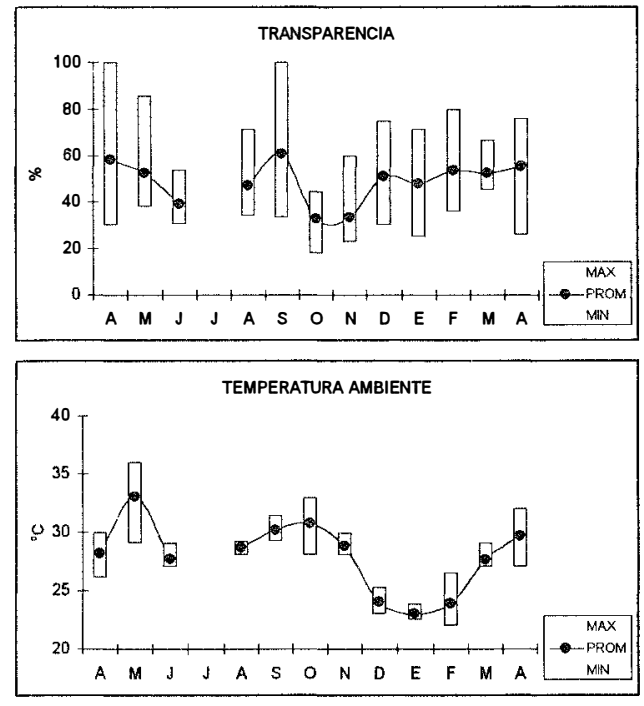

Fig.3. Variación temporal de los parámetros de temperatura ambiente y transparencia en el sistema Candelaria-Panlau. Se presentan los valores mínimo, máximo y promedio por mes.
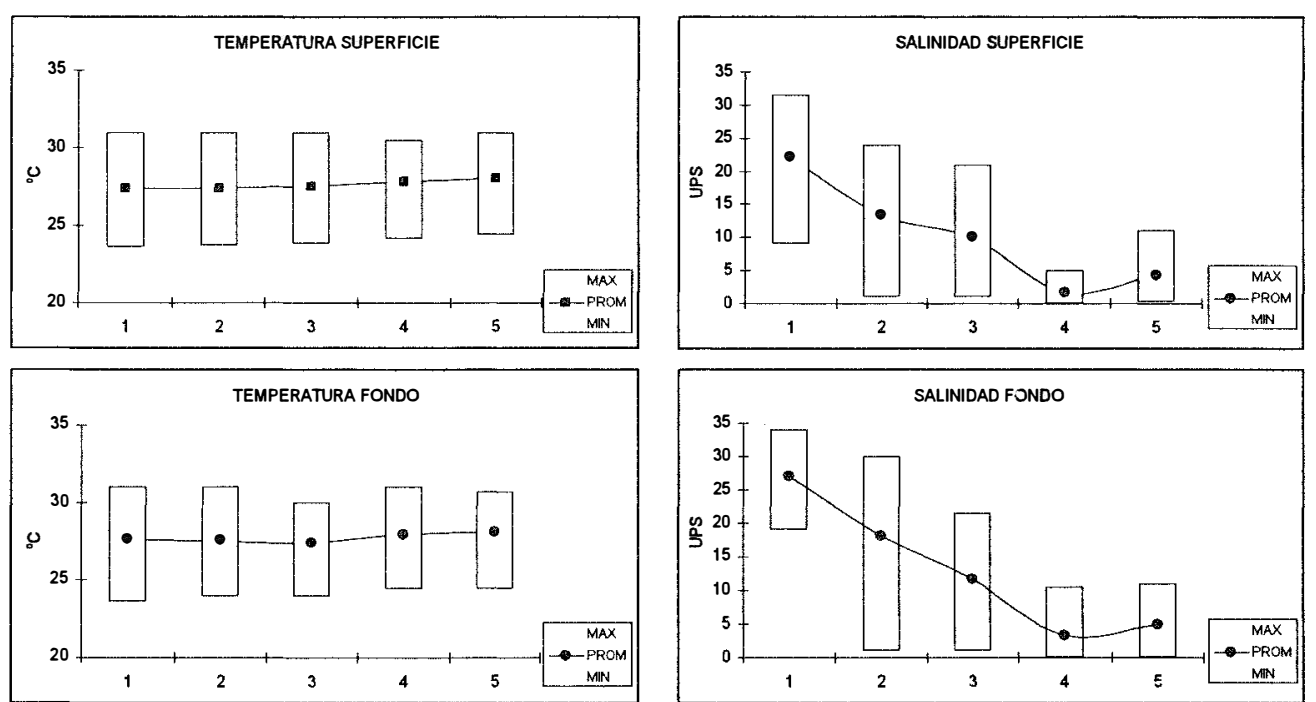

Fig.4. Variación espacial de los parámetros de temperatura y salinidad en el sistema Candelaria-Panlau. Se presentan los valores mínimo, máximo y promedio por estación de muestreo.
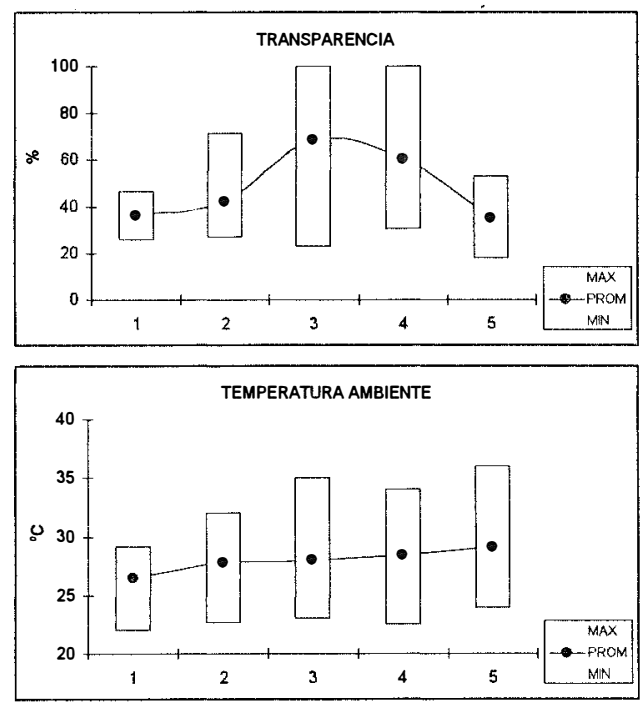

Fig.5. Variación espacial de los parámetros de temperatura ambiente y transparencia en el sistema Candelaria-Panlau. Se presentan los valores mínimo, máximo y promedio por estación de muestreo. 
La comunidad de peces en el sistema Candelaria-Panlau quedó representada por 52 especies (Cuadro 1), con un total de 7926 organismos, $144.7 \mathrm{~kg}$ de peso, una densidad de $0.09 \mathrm{ind} / \mathrm{m}^{2}$, biomasa de $1.61 \mathrm{~g} / \mathrm{m}^{2}$ y peso promedio de 18.25 . g/ind, el índice de diversidad (H'n) fue de 1.879 , equitatividad $\left(\mathrm{J}^{\prime}\right)$ de 0.475 y riqueza de especies (D') de 5.681 .

\section{CUADRO 1}

Listado sistemático de las especies de peces capturadas en el sistema Candelaria-Panlau

Familia

Especie

\begin{tabular}{|c|c|c|}
\hline \multirow{2}{*}{$\begin{array}{l}\text { Dasyatidae } \\
\text { Engraulidae }\end{array}$} & 1. & Dasyatis sabina (Le Sueur 1824) \\
\hline & 2. & $\begin{array}{l}\text { Anchoa mitchilli (Hildebrand } \\
\text { 1943) } \\
\text { Cetengraulis edentulus (Cuvier } \\
\text { 1929). }\end{array}$ \\
\hline Clupeidae & 4. & $\begin{array}{l}\text { Dorosoma petenense (Günther } \\
\text { 1866) }\end{array}$ \\
\hline Ariidae & $\begin{array}{l}5 . \\
6 . \\
7 .\end{array}$ & $\begin{array}{l}\text { Bagre marinus (Mitchill 1815) } \\
\text { Arius felis (Linnaeus 1766) } \\
\text { Cathorops melanopus (Günther } \\
\text { 1864) }\end{array}$ \\
\hline Synodontidae & 8. & Synodus foetens (Linnaeus 1766) \\
\hline Batrachoididae & 10. & $\begin{array}{l}\text { Porichthys porossissimus } \\
\text { (Valenciennes 1837) } \\
\text { Opsanus beta (Goode \& Bean } \\
\text { 1882) }\end{array}$ \\
\hline Syngnathidae & 11. & $\begin{array}{l}\text { Syngnathus louisianae (Günther } \\
1870 \text { ) }\end{array}$ \\
\hline Triglidae & 12. & $\begin{array}{l}\text { Prionotus carolinus (Linnaeus } \\
\text { 1766) } \\
\text { P. punctatus Bloch } 1793\end{array}$ \\
\hline Centropomidae & 14. & $\begin{array}{l}\text { Centropomus parallelus (Poey } \\
1860 \text { ) }\end{array}$ \\
\hline Carangidae & $\begin{array}{l}16 . \\
17 . \\
18 .\end{array}$ & $\begin{array}{l}\text { Chloroscombrus chrysurus } \\
\text { (Linnaeus 1766) } \\
\text { Vomer setapinnis (Mitchill 1814) } \\
\text { Caranx crysos (Mitchil 1815) } \\
\text { Hemicaranx amblyrhynchus } \\
\text { (Cuvier 1833) }\end{array}$ \\
\hline Lutjanidae & 19. & Lutjanus griseus (Linnaeus 1758) \\
\hline \multirow[t]{2}{*}{ Gerreidae } & $\begin{array}{l}20 . \\
21 .\end{array}$ & $\begin{array}{l}\text { Diapterus auratus (Ranzani } 1842 \\
\text { D. rhombeus (Cuvier \& } \\
\text { Valenciennes } 1829 \text { ) }\end{array}$ \\
\hline & 22. & $\begin{array}{l}\text { Eucinostomus argenteus } \text { Baird } \\
\& \text { Girard } 1854\end{array}$ \\
\hline
\end{tabular}

23. E. gula (Cuvier \& Valenciennes 1830)

24. E. melanopterus(Bleecker 1863)

25. Eugerres plumieri (Cuvier 1830)

Haemulidade

26. Orthopristes chrysoptera (Linnaeus 1766)

Sparidae

27. Archosargus probatocephalus (Walbaum 1792)

28. A. rhomboidalis (Linnaeus 1758)

Polynemidae

29. Polydactylus octonemus Girard 1858

Sciaenidae

30. Bairdiella chrysura (Lacepede 1803)

31. B. ronchus (Cuvier \& Valenciennes 1830)

32. Cynoscion nebulosus (Cuvier 1830)

33. C. arenarius Ginsburg 1929

34. Micropogonias undulatus (Linnaeus 1766)

35. Menticirrhus americanus (Linnaeus 1758)

36. Stellifer lanceolatus Holbrook 1855

37. Odontoscion dentex (Cuvier 1830)

Cichlidae 38. Cichlasoma urophthalmus (Günther 1862)

39. Cichlasoma spp

Gobiidae 40. Gobionellus hastatus Girard 1858

41. Gobiosoma bosci (Lacepede 1758)

Ephippidae 42. Chaetodipterus faber (Broussonet 1782)

Trichiuridae

43. Trichiurus lepturus (Linnaeus 1758)

Bothidae 44. Citharichthys spilopterus Günther 1862

Soleidae 45. Achirus lineatus (Linnaeus 1758)

46. Trinectes maculatus (Bloch \& Schneider 1801)

Tetraodontidae 47. Sphoeroides testudineus (linnaeus 1743)

48. S. nephelus (Goode \& Bean 1758)

49. S. spengleri (Bloch, 1785)

Diondontidae 50. Chilomicterus schoepfi (Walbaum 1792)

En la escala temporal, la densidad y la biomasa presentan valores promedio más altos en abril $\left(0.17 \mathrm{ind} / \mathrm{m}^{2}\right.$ y $3.5 \mathrm{~g} / \mathrm{m}^{2}$ respectivamente) 
y más bajos en febrero $\left(0.033 \mathrm{ind} / \mathrm{m}^{2}\right.$ y 0.57 $\left.\mathrm{g} / \mathrm{m}^{2}\right)$. El peso promedio osciló entre $30.4 \mathrm{~g} /$ ind en agosto y $10.8 \mathrm{~g}$ /ind en diciembre (Fig. 6). Los indicadores de diversidad muestran la siguiente variación: índice de diversidad H'n entre 1.073 en diciembre y 2.037 en agosto, equitatividad $\left(\mathrm{J}^{\prime}\right)$ entre 0.338 en diciembre y 0.666 en marzo, y riqueza de especies (D') entre 2.167 en septiembre y 4.472 en octubre (Fig.7).
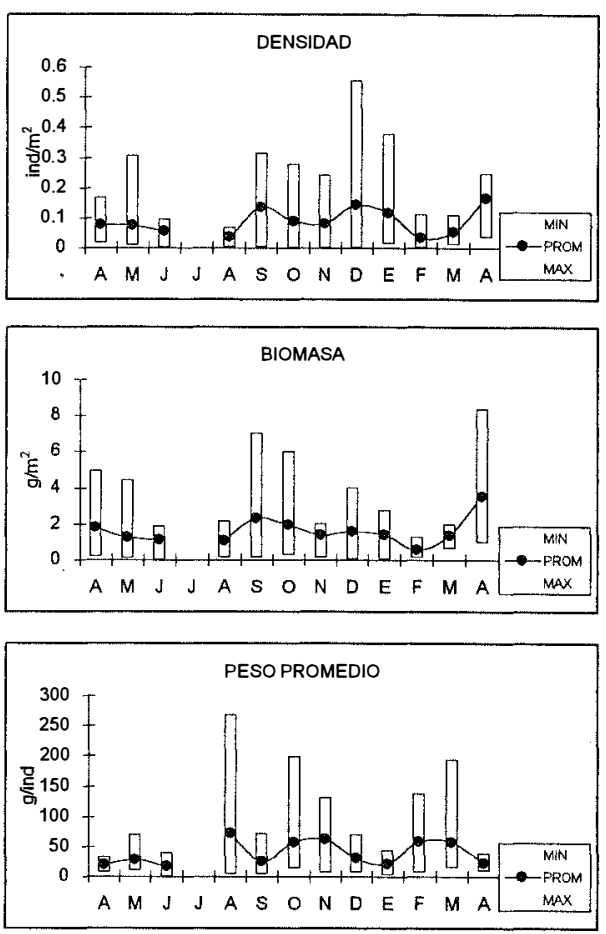

Fig.6. Variación temporal de los parámetros de abundancia de la comunidad de peces en el sistema Candelaria-Panlau. Se presentan los valores mínimo, máximo y promedio por mes.

En escala espacial, los valores promedio de diversidad y abundancia presentan los siguientes intervalos: densidad entre 0.04 y 0.17 $\mathrm{ind} / \mathrm{m}^{2}$, biomasa entre 1.33 y $2.41 \mathrm{~g} / \mathrm{m}^{2}$, peso promedio entre 8.7 y $34.6 \mathrm{~g} /$ ind (Fig. 8), índice de diversidad H'n entre 1.253 y 2.078 , equitatividad $\left(\mathrm{J}^{\prime}\right)$ entre 0.405 y 0.585 y riqueza de especies (D') entre 3.085 y 5.116 (Fig. 9).
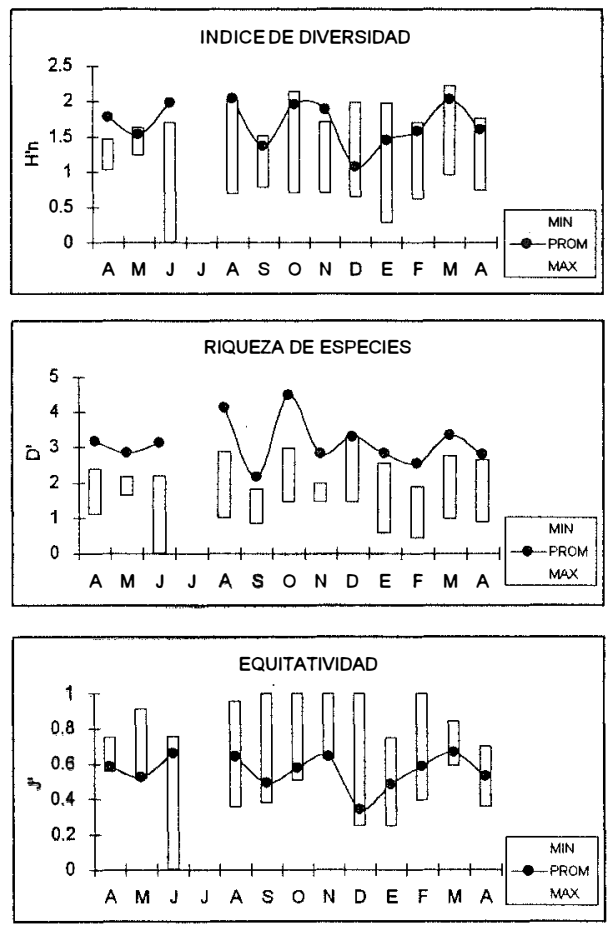

Fig.7. Variación temporal de los parámetros de diversidad de la comunidad de peces en el sistema Candelaria-Panlau. Se presentan los valores mínimo, máximo y promedio por mes.

Bajo el análisis integrado de los parámetros de abundancia, distribución y frecuencia de aparición, las especies con dominio ecológico en el sistema son: Cathorops melanopus, Diapterus rhombeus, Anchoa mitchilli, Sphoeroides testudineus, Bairdiella chrysura, B. ronchus, Cynoscion arenarius, y $C$. nebulosus, que en conjunto representan el $90.9 \%$ en número y el $78.9 \%$ en peso de la captura total.

El análisis de factores con el método de extracción de componentes principales se representó con la rotación de varianza máxima normalizada. En todos los casos la varianza porcentual acumulada para dos factores fue superior al $90 \%$, de manera que la representación se hace en dos planos. Para el caso de la matriz de parámetros ambientales por estación y por mes, el factor uno alcanzó un eigenvalor de 7.8 con una varianza total del $87.7 \%$, y el factor dos 
con un eigenvalor de 0.7 y $7.6 \%$ de varianza total, los componentes de esta matriz con mayor valor en el factor dos son la salinidad de superficie y de fondo. En este mismo sentido la matriz de número de individuos por especie y por estación muestra los siguientes eigenvalores: 3.9 para el factor uno y 0.6 para el factor dos con una varianza total de $78.6 \%$ y $12.5 \%$ respectivamente, el valor más altos para el factor dos lo presenta la estación cinco (Fig. 10).
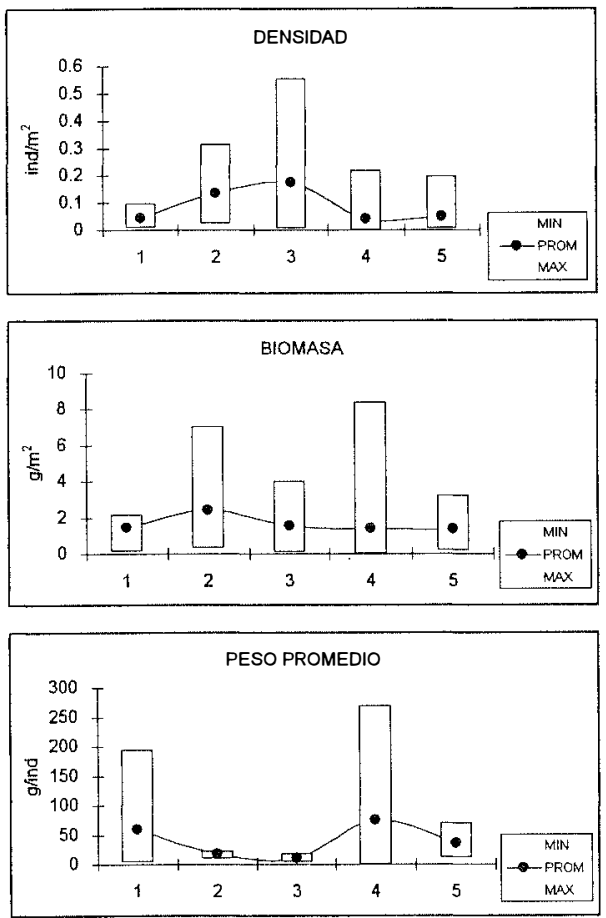

Fig.8. Variación espacial de los parámetros de abundancia de la comunidad de peces en el sistema Candelaria-Panlau. Se presentan los valores mínimo, máximo y promedio por estación de muestreo.

En escala temporal, la matriz de número de individuos por especie y por mes presenta un eigenvalor de 9.1 con $75.6 \%$ de varianza total para el factor uno y un eigenvalor de 1.2 con 10.7 $\%$ de varianza total para el factor 2 , los valores que se desagregan del conjunto son febrero, octubre, agosto y junio. Finalmente, la matriz de número de individuos por especie y por estación transpuesta presenta los eigenvalores siguientes: para el factor uno de 41.2 con $79.2 \%$ de varianza total y para el factor dos de 3.3 con $6.4 \%$ de varianza total, destacan los valores de: Diapterus rhombeus, Cathorops melanopus, Bardiella chrysura, B. ronchus y Anchoa mitchilli (Fig. 10).
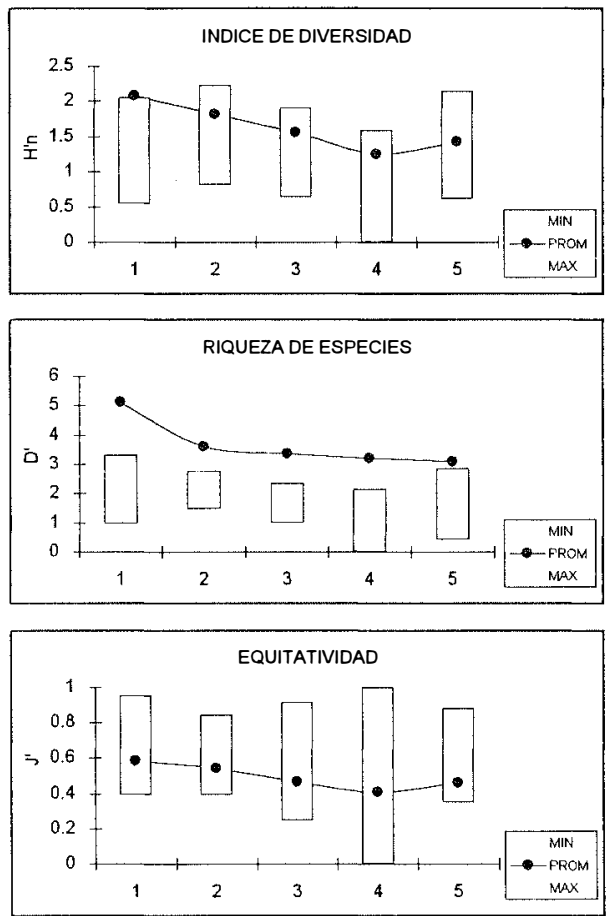

Fig.9. Variación espacial de los parámetros de diversidad de la comunidad de peces en el sistema Candelaria-Panlau. Se presentan los valores mínimo, máximo y promedio por estación de muestreo.

\section{DISCUSION}

De acuerdo con lo reportado por YáñezArancibia y Day (1988), Fuentes-Yaco (1990) y Avilés-Alatriste (1991) el comportamiento ambiental espacial y temporal en la región esta condicionado básicamente por la ubicación geográfica latitudinal, la descarga de los ríos, la marea, la geomorfología y la circulación litoral. Así, la temperatura tanto ambiental como del agua en el sistema Candelaria-Panlau es máxima en mayo y mínima en enero como respuesta 
Análisis de Parámetros Ambientales

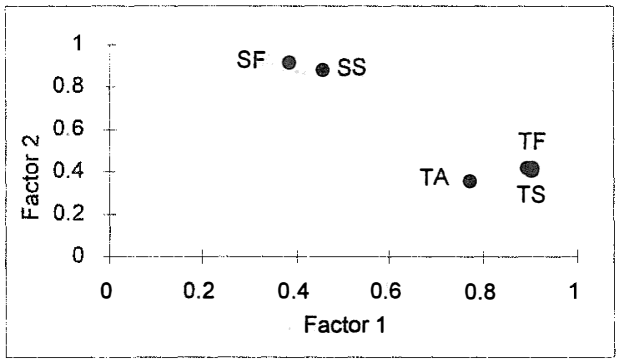

Análisis por Meses

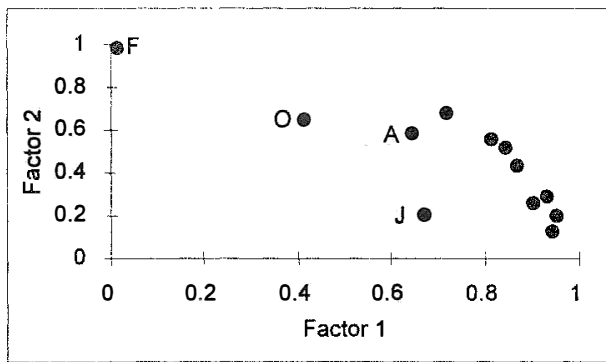

Análisis por Estaciones de Muestreo

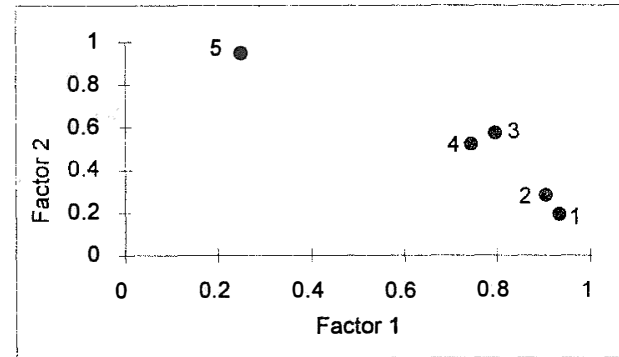

Análisis por Especies

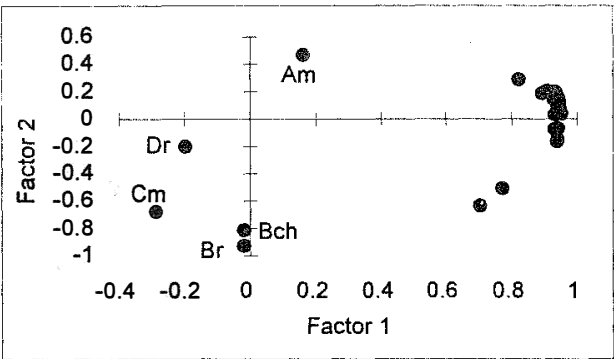

Fig.10. Análisis de factores por el método de extracción de componentes principales de las matrices de parámetros ambientales, de abundancia numérica de la comunidad de peces en escalas espacial, temporal y por especie. $\mathrm{SS}=$ salinidad de superficie, $\mathrm{SF}=$ salinidad de fondo, $\mathrm{TS}=$ temperatura de superficie, $\mathrm{TF}=$ temperatura de fondo, $\mathrm{TA}=$ temperatura ambiente $\mathrm{F}=$ febrero, $\mathrm{O}=$ octubre, $\mathrm{A}=\mathrm{Agosto}, \mathrm{J}=$ junio, $\mathrm{Am}=$ Anchoa mitchilli, $\mathrm{Dr}=$ Diapterus rhombeus, $\mathrm{Cm}=$ Cathorops melanopus, $\mathrm{Br}=$ Bairdiella ronchus y $\mathrm{Bch}=$ Bairdiella chrysura.

a la estacionalidad de primavera e invierno. De la misma forma, la salinidad refleja el efecto del esfuerzo combinado de la marea y de la descarga de los ríos. La temporada de lluvias en la región es entre julio y octubre y la mayor descarga de los ríos se observa justamente en noviembre como una respuesta con retraso, lo que se refleja directamente en la salinidad.

La comunidad de peces del sistema Candelaria-Panlau, representada por un total de 52 especies e integrada a la información existente para otros sistemas fluvio-lagunares, Laguna de Términos y Sonda de Campeche, permite comparar la magnitud del intercambio ecológico entre estos ecosistemas. Ayala-Pérez et al. (1993) reporta 41 especies de peces para el sistema PomAtasta, 64 para Palizada-Del Este y 50 para Chumpam-Balchacah, en conjunto se integran 81 especies. De las 52 especies identificadas en el sistema Candelaria-Panlau el $90 \%$ se han registrado en los otros sistemas fluvio-lagunares.
Prionotus punctatus, Hemicaranx amblyrhynchus, Odontoscion dentex, Sphoeroides spenglery y Chilomicterus schoepfi, son las especies propias de Candelaria-Panlau.

Para la Laguna de Términos y Sonda de Campeche se han reportado 122 y 149 especies respectivamente (Yáñez-Arancibia et al. 1985), entre las cuales se encuentra el $94 \%$ de las especies de Candelaria-Panlau, las especies diferentes son Dorosoma petenense, Vomer setapinnis y Hemicaranx amblyrhynchus.

Al comparar los valores de biomasa y peso promedio reportados para la Sonda de Campeche y Laguna de Términos con los observados en este estudio, es posible proporcionar argumentos a favor de la hipótesis de que los sistemas fluvio-lagunares son hábitats utilizados por una diversa comunidad de peces para actividades de crianza, crecimiento y alimentación (Vera-Herrera et al. 1988b), así, Yáñez-Arancibia et al. (1988a) reporta para la Sonda de 
Campeche valores de biomasa de $0.62 \mathrm{~g} / \mathrm{m}^{2}$ y de peso promedio de $38.2 \mathrm{~g} / \mathrm{ind}$, del mismo modo Yáñez-Arancibia et al. (1988b) para la Laguna de Términos puntualiza valores de $1.54 \mathrm{~g} / \mathrm{m}^{2}$ y $35.2 \mathrm{~g} / \mathrm{ind}$ y finalmente para Candelaria-Panlau los valores fueron $1.61 \mathrm{~g} / \mathrm{m}^{2}$ y $18.2 \mathrm{~g} /$ ind respectivamente. Estas tendencias, permiten observar que los sistemas fluvio-lagunares en particular Candelaria-Panlau son usados en preferencia por abundantes organismos de tallas pequeñas.

Por otra parte, de las ocho especies con dominancia ecológica, tres son netamente estuarinas y el resto son marinas con dependencia estuarina, lo cual refleja nuevamente el importante nivel de intercambio con la zona marina adyacente.

Por el análisis de factores de las matrices de parámetros ambientales y de parámetros ecológicos, es posible discutir de manera integrada la información que hasta aquí se ha presentado. En primer lugar, al analizar los parámetros ambientales tanto en escala espacial como temporal se identifica a la salinidad como el componente más importante, esto permite inferir que este parámetro es el que condiciona en mayor medida el comportamiento ambiental del sistema. Tal y como se ha mencionado anteriormente, el volumen de descarga de los ríos y la marea son las principales funciones de fuerza en los sistemas fluvio-lagunares y es la salinidad, entre los parámetros observados, la que refleja de mejor forma esta dinámica.

En el análisis de las matrices de número de individuos por especie por estación y número de individuos por especie por mes, se destacan los valores de la estación cinco y del mes de febrero. La estación cinco se ubica en la desembocadura del río Mamantel en donde el intervalo de salinidad es de 0.0 a 11.0 UPS y en promedio es la segunda estación menos salina después de la estación cuatro ubicada en la desembocadura del río Candelaria. Esta condición le confiere a este sitio características particulares que tienen un reflejo importante en la distribución y abundancia de la comunidad de peces. Por otra parte, el mes de febrero que es la parte final de la época de nortes, presenta aportes pluviales y fluviales abundantes, lo que se reflejan en la condición de baja salinidad, transparencia y por consiguiente en los parámetros ecológicos de la comunidad de peces. En este nivel de análisis es importante resaltar que estos resultados permiten plantear nuevas hipótesis.

Finalmente, en el análisis de la matriz transpuesta por especies, se confirma la definición de las especies con dominancia ecológica, ya que los valores que se desagregan corresponden a las especies previamente identificadas bajo este concepto.

Ya se ha mencionado que los sistemas fluvio-lagunares asociados a la Laguna de Términos, constituyen reservorios naturales de gran valor ecológico que tienen que ser objeto de un adecuado manejo tanto para el uso de sus recursos como para su conservación, de tal forma que información como la que aquí se ha presentado, integrada a una perspectiva regional, deben fortalecer los criterios para la toma de decisiones.

\section{RESUMEN}

Se describe el comportamiento de los parámetros de temperatura, salinidad, transparencia del agua y temperatura ambiente, y de la abundancia y diversidad de la comunidad del peces del sistema Candelaria-Panlau en escalas temporal y espacial. Los trabajos de campo se realizaron de manera mensual en cinco estaciones entre abril de 1993 y abril de 1994. La comunidad de peces quedó representada por 50 especies con 7926 organismos y $144.7 \mathrm{~kg}$ de peso. Los valores de abundancia y diversidad de la comunidad fueron: $0.09 \mathrm{ind} / \mathrm{m}^{2}, 1.61 \mathrm{~g} / \mathrm{m}^{2} 18.25 \mathrm{~g} / \mathrm{ind}, \mathrm{H} \cdot \mathrm{n}=1.879, \mathrm{~J}=0.475$ y $\mathrm{D}=5.681$. Las especies con dominio ecológico fueron: Cathorops melanopus, Diapterus rhombeus, Anchoa mitchilli, Sphoeroides testudineus, Bairdiella chrysura, B. ronchus, Cynoscion arenarius, y C. nebulosus, que en conjunto representan el $90.9 \%$ en número y el $78.9 \%$ en peso de la captura total. Las matrices de valores de los parámetros ambientales y de abundancia numérica de la comunidad de peces, fueron sometidas a un análisis de factores con el método de extracción de componentes principales, su representación gráfica confirma la definición de especies dominantes y demuestra que la salinidad, estación cinco y mes de febrero son los componentes que caracterizan al sistema y a la comunidad de peces.

\section{AGRADECIMIENTOS}

A Francisco Vera-Herrera, Hernán Alvarez Guillen, Andrés Reda Deara de la Estación El Carmen, Instituto de Ciencias del Mar y Limnología, 
UNAM, y a Arturo Aguirre-León del Departamento El Hombre y su Ambiente de la Universidad Autónoma Metropolitana Xochimilco.

\section{REFERENCIAS}

Aguirre-León, A., I. Cabrera-Díaz, A. Bernal-Becerra \& L.A. Ayala-Pérez. 1995. Ecología y evaluación de los recursos pesqueros asociados al sistema fluvio-lagunar Pom-Atasta, Campeche. Inf. Tec. Depto. El Hombre y su Ambiente, Universidad Autónoma Metropolitana Xochimilco, México, D.F., 130 p.

Alvarez Del Villar, J. 1970. Peces Mexicanos (claves). Instituto Nacional de Investigaciones Biológico Pesqueras. Comisión Nacional Consultiva de Pesca. SIC, México, D.F. 166 p.

Amezcua-Linares, F. \& A. Yáñez-Arancibia. 1980. Ecología de los sistemas fluvio-lagunares asociados a la Laguna de Términos. El hábitat y estructura de las comunidades de peces. An. Centro Cienc. del Mar y Limnol. Universidad Nacional Autónoma de México. México, D.F. 7: $68-118$.

Anónimo 1976. Volumen de descarga del río Candelaria. Secretaría de Recursos Hidráulicos, México, D.F. 587 p.

Anónimo 1994a. Decreto por el que se declara como área natural protegida con el carácter de protección de flora y fauna, la región conocida como Laguna de Términos, ubicada en los municipios de Carmen, Palizada y Champotón, Estado de Campeche. Diario Oficial de la Federación, Gobierno Constitucional de los Estados Unidos Mexicanos, Tomo 489 No. $4.58-65$ p.

Anónimo 1994b. Tablas de predicción de mareas: Datos geofísicos serie A (oceanografía). Instituto de Geofísica. Universidad Nacional Autónoma de México, México, D.F. $191 \mathrm{p}$.

Avilés-Alatriste, O.A. 1991. Recopilación y procesamiento primario de información estadística de registros meteorológicos y captura pesquera para el sistema ChumpamBalchacah (Sur de Golfo de México). Inf. Servicio Social. División de Ciencias Biológicas y de la Salud, Universidad Autónoma Metropolitana Xochimilco, México, D.F. 24 p.

Ayala-Pérez, L.A. 1989. Ecología y características poblacionales de dos especies de peces dominantes en el sistema estuarino Palizada-Del Este, sur del Golfo de México: Anchoa mitchilli (Engraulidae) y Petenia splendi$d a$ (Cichlidae). Tesis de Maestría en Ciencias del Mar Inst. Cienc. del Mar y Limnol. Universidad Nacional Autónoma de México, México, D.F. 112 p.

Ayala-Pérez, L.A., A. Aguirre-León, O.A. Avilés-Alatriste, M.T. Barreiro-Güemes \& J.L. Rojas-Galavíz. 1993. Peces de sistemas fluvio-lagunares, Laguna de Términos,
Campeche. p. 596-608. In: Salazar-Vallejo S.I. \& N.E. González (eds.). Biodiversidad Marina y Costera de México. Com. Nal. Biodiversidad - Centro de Iinvestigaciones de Quintana Roo, México.

Ayala-Pérez, L.A., A. Pérez-Velázquez, A. Aguirre-León \& S. Díaz-Ruiz. 1995. Abundancia nictemeral de corvinas (Pisces : Sciaenidae) en un sistema costero del sur del Golfo de México. Hidrobiológica 5: 37-44.

Ayala-Pérez, L.A., J.L. Rojas-Galavíz \& O.A. Avilés-Alatriste. 1996. Crecimiento, reproducción y dinámica poblacional de Anchoa mitchilli (Pisces: Engraulidae) en el sistema Palizada-Del Este, Campeche, México. Rev. Biol. Trop. 44/45: 507-518.

Castro-Aguirre, J.L. 1978. Catálogo sistemático de los peces marinos que penetran a las aguas continentales de México con aspectos zoogeográficos y ecológicos. Inst. Nal. Pesca México, Serie Científica 19. 298 p.

Fischer, W. (Ed.). 1978. Species identification sheets for fishery purposes. Western Central Atlantic (Fishing area 31). FAO, Roma. 897 p.

Fuentes-Yaco, C. 1990. Dinámica hidrológica y análisis de parámetros físico-químicos en el ecosistema fluvio-deltáico-lagunar-estuarino del río Palizada, Campeche. Tesis de Maestría en Ciencias del Mar. Inst. Cienc. del Mar y Limnól. Universidad Nacional Autónoma de México, México, D.F.

Gauch, H.G. Jr. 1989. Multivariate analysis in community ecology. Cambridge University, Nueva York. 298 p.

Hildebrand, S.F. 1943. American anchovies (Fam. Engraulidae). Bull. Binham Oceanogr. Coll. 8. 165 p.

Hoese, H.D. \& R.S. Moore. 1977. Fishes of the Gulf of Mexico, Texas, Louisiana and Adjacent Waters. Texas A\&M University, Austin, Texas. 376 p.

Jordan, D.S. \& B.W. Evermann. 1886-1900. The fish of north and middle America. Bull. Nat. Mus. USA. 3313 p

Ludwig, J.A. \& J.F. Reynolds. 1988. Statistical ecology. A primer on methods and computing. Wiley. Nueva York. $337 \mathrm{p}$.

Magurran, A.E. 1988. Ecological diversity and it's measurement. Princenton University, Princeton. $179 \mathrm{p}$.

Margalef, R. 1969. Perspectives in ecological theory. Chicago University, Chicago, Ilinois. $111 \mathrm{p}$.

Morales, C.J. 1986. Estudio sistemático y ecológico de la ictiofauna de la Laguna del Vapor, Campeche. Tesis de Licenciatura en Biología. Escuela Nacional de Ciencias Biológicas. Instituto Politécnico Nacional. México, D.F.

Nelson, J.S. 1994. Fishes of the World. Wiley. Nueva York. $465 \mathrm{p}$. 
Nitsch, S.L. 1992. Ictioecología del sistema fluvio-lagunar Candelaria-Panlau asociado a la Laguna de Términos, Campeche (sur del Golfo de México). Tesis de Licenciatura en Biología. Escuela Nacional de Estudios Profesionales Iztacala, Universidad Nacional Autónoma de México, México, D.F.

Pielou, E.C. 1966. The measurement of diversity in different types of biological collections. J. Theoret. Biol. 13: $131-144$.

Pielou, E.C. 1984. The interpretation of ecological data. A primer on classification and ordination. Wiley, Nueva York. $263 \mathrm{p}$.

Reséndez, A. 1981a. Estudio de los peces de la Laguna de Términos, Campeche, México. I. Biótica 6: 239-291.

Reséndez, A. 1981b. Estudio de los peces de la Laguna de Términos, Campeche, México. II. Biótica 6: 345-430.

Rojas-Galavíz, J.L., F. Vera-Herrera, A. Yáñez-Arancibia \& J.W. Day Jr. 1988. The Usumacinta/Palizada river delta as ecosystem. Inf. Téc. CONACyT. Clave PCCNCNA031524 Inst. Cienc. del Mar y Limnól. Universidad Nacional Autónoma de México, México, D.F. 160-184 p.

Shannon, C.E. \& W. Weaver. 1963. The mathematical theory of communication. University of Illinois, Chicago, Ilinois. $119 \mathrm{p}$.

Soberón-Chávez, G. 1984. Mecanismos de producción de las poblaciones de peces demersales de la plataforma continental del sur del Golfo de México: Variables de interacción ecológica. Tesis de Maestría en Ciencias del Mar. Instituto de Ciencias del Mar y Limnología. Universidad Nacional Autónoma de México, México, D.F.

Vera-Herrera, F., J.L. Rojas-Galavíz \& A. Yañez-Arancibia. 1988a. Pantanos dulceacuícolas influenciados por la marea en la región de la Laguna de Términos: Estructura ecológica del sistema fluvio-deltáico del río Palizada. Proceedings of the Simposium on the Ecology and Conservation of the Usumacinta-Grijalva Delta. INIREB Tabasco, WWF Brehm Fonds, IUCN, ICT, Gob. Estado de Tabasco, México. 383-402 p.

Vera-Herrera, F., J.L. Rojas-Galavíz, C. Fuentes-Yaco, L.A. Ayala-Pérez, H. Álvarez-Guillen \& C. Colorado-Molina. 1988b. Descripción ecológica del sistema fluvio-lagunar deltáico del río Palizada. p. 51-88. In: A. Yáñez-Arancibia \& J.W. Day Jr. (eds.). Ecología de los ecosistemas costeros en el sur del Golfo de México: la Región de la Laguna de Términos. Instituto de Ciencias del Mar y Limnología, Coast. Ecol. Inst. LSU. Editorial Universitaria. Universidad Nacional Autónoma de México, México, D.F.

Yáñez-Arancibia, A. \& J.W. Day Jr. 1988. Ecological characterization of Terminos Lagoon, a tropical lagoon-estuarine system in the southern Gulf of Mexico. p. 1-26. In: A. Yáñez-Arancibia \& J.W. Day, Jr. (eds.). Ecología de los ecosistemas costeros en el sur del Golfo de México: La región de la Laguna de Términos. Instituto de Ciencias del Mar y Limnología, Coast. Ecol. Inst. LSU. Editorial Universitaria. Universidad Nacional Autónoma de México, México, D.F.

Yáñez-Arancibia, A., A. Lara-Domínguez, P. Sánchez-Gil\& H. Alvarez-Guillén, 1988a. Evaluación ecológica de las comunidades de peces de la Laguna de Términos y Sonda de Campeche, p. 323-356. In A. Yáñez-Arancibia \& J.W. Day Jr. (eds.). Ecología de los ecosistemas costeros en el sur del Golfo de México: La región de la Laguna de Términos. Instituto de Ciencias del Mar y Limnología. Universidad Nacional Autónoma de México, Coast. Ecol. Inst. LSU. México, D.F.

Yáñez-Arancibia, A., A.L. Lara-Domínguez, P. SánchezGil, H. Álvarez-Guillén, G. Soberón-Chávez \& J.W. Day, Jr., 1988b. Dinámica de las comunidades nectónicas costeras en el sur del Golfo de México, p. 357-380. In A. Yáñez-Arancibia \& J.W. Day Jr. (eds.). Ecología de los ecosistemas costeros en el sur del Golfo de México: La región de la Laguna de Términos Instituto de Ciencias del Mar y Limnología. Universidad Nacional Autónoma de México, Coast. Ecol. Inst. LSU. México, D.F.

Yáñez-Arancibia, A., A.L. Lara-Domínguez, P. SánchezGil, I. Vargas-Maldonado, M.C. García-Abad, H. Álvarez-Guillén, M. Tapia-García, D. Flores-Hernández \& F. Amezcua-Linares. 1985. Ecology and evaluation of fish community in coastal ecosystem: estuary shelf interrelationship in the southern Gulf of Mexico, p. 475-498. In A. Yáñez-Arancibia (ed.). Fish community ecology in estuaries and coastal lagoons: Towards and ecosystem integration. Editorial Universitaria. Universidad Nacional Autónoma de México. México, D.F. 